\title{
The design of an ECR plasma system and its application to InP grown by CBE
}

\author{
M.E. Sherwin, G.O. Munns, E.G. Woelk, T.J. Drummond *, M.E. Elta, F.L. Terry \\ and G.I. Haddad \\ Center for High Frequency Micro-Electronics, 2435 EECS Building, The University of Michigan, Ann Arbor, Michigan 48109-2122, USA
}

\begin{abstract}
An electron cyclotron resonance (ECR) plasma system has been designed for the purpose of using an excited beam of gases during $\mathrm{CBE}$ growth. The system was designed to use hydrogen, nitrogen and argon. An ECR plasma system has the ability to ignite a low pressure and low temperature plasma with very low ion energies, which should minimize any damage to the growing layer. The motivation behind using a plasma during growth is the ability of atomic hydrogen to remove contaminants from the growing layer and to enhance the decomposition of organometallic precursors at low substrate temperatures. InP grown with a hydrogen plasma showed an n-type background carrier concentration of $6.0 \times 10^{16} \mathrm{~cm}^{-3}$, with a rough surface and a strong photoluminescence peak at $1.378 \mathrm{eV}$. A control sample grown with excess hydrogen but without the plasma had a background carrier concentration of $1.0 \times 10^{15}$ $\mathrm{cm}^{-3}$, a $77 \mathrm{~K}$ mobility of $65,000 \mathrm{~cm}^{2} / \mathrm{V} \cdot \mathrm{s}$ and a very weak photoluminescence peak at $1.378 \mathrm{eV}$. The most likely cause for the layer degradation during plasma growth is an intrinsic defect such as an antisite defect or a vacancy. The n-type nature of the layer and the relatively high carrier concentration would seem to exclude the possibility of carbon or any other unintentional impurities.
\end{abstract}

\section{Introduction}

Hydrogen and nitrogen plasmas have been used to aid in the growth of $\mathrm{GaAs}, \mathrm{GaN}$ and other materials [1-3]. When the epitaxial process occurs at very low pressures, below $1 \times 10^{-4}$ Torr, an ECR plasma source is one of the most convenient techniques to generate a plasma. An ECR plasma system is a powerful method for generating an excited beam of gases during CBE growth.

ECR plasmas have been proven effective for the growth of nitrides, specifically GaN and AlN. For nitride growth without plasma assistance, it was found that hydrazine is a better source of nitrogen than ammonia [4]. However, work done with ECR plasmas and nitride growth have shown that nitrogen can be an effective source material [5]. The reactivity of ionized nitrogen eases some of the constraints on the growth conditions. The substrate temperature is no longer limited by the

\footnotetext{
* Present address: Sandia National Laboratories, Albuquerque, New Mexico 87185, USA.
}

decomposition temperature of the group $\mathrm{V}$ precursor. Meikle et al. [5] have suggested that the addition of hydrogen to a nitrogen plasma should help prevent the formation of $\mathrm{Al}-\mathrm{C}$ during the growth of AlN from trimethylaluminum. In designing an ECR system for CBE growth of nitrides, it is important to have the ability to arbitrarily vary the composition of the plasma.

The ECR plasma injector was installed in a first generation Varian Gen II MOMBE reactor. The source materials used were trimethylindium (TMI) and 100\% phosphine. The CBE system has been described elsewhere [6]. The experiments were carried out with a TMI flow rate of 1.25 SCCM, a $\mathrm{V} / \mathrm{III}$ ratio of 14.2 , a substrate setpoint of $580^{\circ} \mathrm{C}$, an alkyl injector temperature of $50^{\circ} \mathrm{C}$, and a hydride injector temperature of $894^{\circ} \mathrm{C}$. The actual substrate temperature is estimated to be $525^{\circ} \mathrm{C}$ [6]. The samples were analyzed with patterned Hall measurements, etching $C-\mathrm{V}$ measurement and photoluminescence (PL). The excitation wavelength for the PL measurements was $488 \mathrm{~nm}$, and the intensity was approximately $1.2 \mathrm{~W} / \mathrm{cm}^{2}$. The $\mathrm{PL}$ measurements were done at $14 \mathrm{~K}$. 


\section{The ECR plasma system}

The plasma injector was purchased from Wavemat, Inc. [7]. The injector was designed to fit in a standard Knudsen cell port in the source flange of the Varian Gen II MOMBE. A schematic of the injector is shown in fig. 1 . The injector was designed to take advantage of the resonance that occurs when microwave energy couples with the resonance frequency of electrons in a static magnetic field [8]. Making use of this resonance allows the plasma to be ignited at low pressures and low temperatures, minimizing damage to the growing layer. The injector was designed to minimize plasma recombination within the discharge region. The materials located near the discharge region are quartz and stainless steel.

The gas delivery system was designed to allow independent control of gas composition and flow rate into the reactor. The present system was developed for use with nitrogen, hydrogen and argon. A layout of the gas delivery system is shown in fig. 2. There are two distinct portions of the gas delivery system, composition control and flow control into the reactor. The composition of the gas stream is controlled by 3 parallel MKS 1259 mass flow controllers, allowing any mixture of hydrogen, nitrogen and argon. The flow rate into the growth chamber is controller by a molecular flow element (MFE). By varying the pressure across the MFE, the flow rate can be varied up to 50 SCCM, with a resolution better than 0.1 SCCM. However, due to the low conductance of the plasma injector itself $\left(3.7 \times 10^{-3} 1 / \mathrm{s}\right)$ [7], at flow rates over 5 SCCM the presence drop of the injector itself is used to control the gas flow. It is postulated that replacing the MFE with a laminar flow element, which can sustain a larger pressure drop across the element, will result in a more controllable flow rate in the 10 to 20 SCCM range.

\section{Results}

In order to test the applicability of a hydrogen plasma during the growth of InP three layers were grown; bulk undoped InP, InP with a hydrogen plasma, and InP with excess hydrogen but no plasma. The electrical properties are summarized

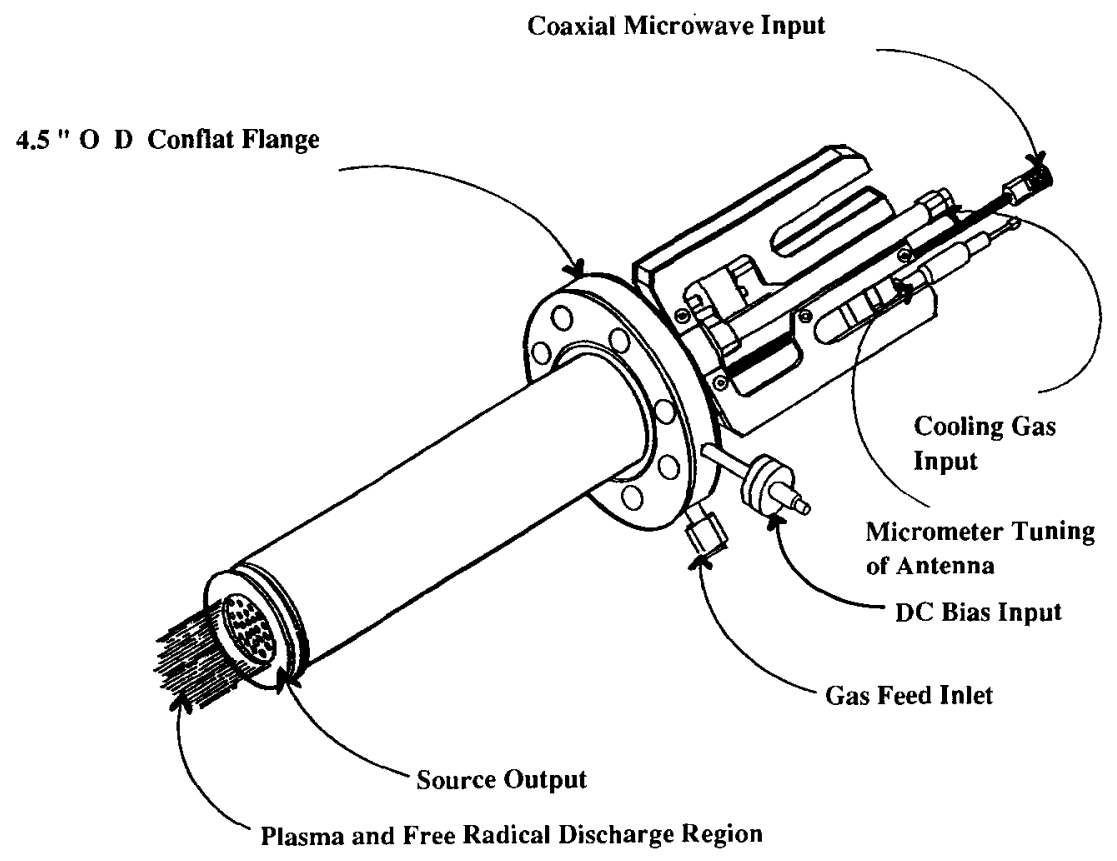

Fig. 1. The ECR plasma injector purchased from Wavemat, Inc. 


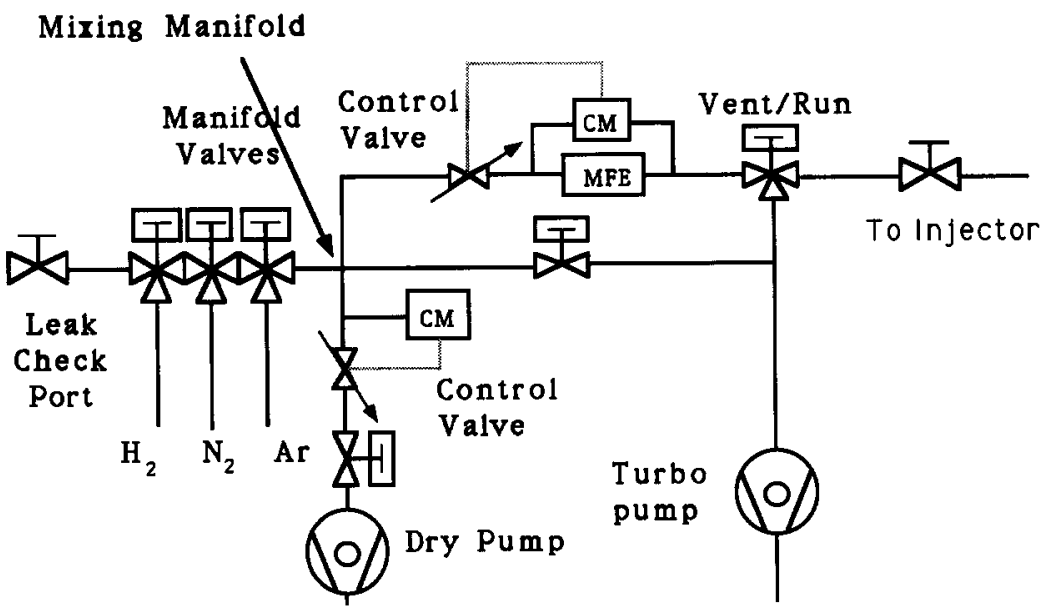

Fig. 2. Layout of the gas delivery system.

in table 1. Samples 135 and 136 had an excess hydrogen flow of $9.5 \mathrm{SCCM}$, while the plasma for sample 135 was ignited with $50 \mathrm{~W}$. Sample 135 showed significant deterioration in surface quality, while samples 134 and 136 had excellent surfaces.

Photoluminescence spectra were taken of the three samples in the 1.2 to $1.5 \mathrm{eV}$ range. Sample 134 shows a band-to-band peak at $1.413 \mathrm{eV}$ with a FWHM of $4.8 \mathrm{meV}$. Sample 135 shows a bandto-band peak at $1.410 \mathrm{eV}$ with a FWHM of 16.8 $\mathrm{meV}$ and a strong second peak at $1.378 \mathrm{eV}$ with a FWHM of $20.7 \mathrm{meV}$. Sample 136 shows a bandto-band peak at $1.413 \mathrm{eV}$ with a FWHM of 4.8 $\mathrm{meV}$ and a weak second peak at $1.378 \mathrm{eV}$. Fig. 3 shows the photoluminescence spectra of samples 134, 135 and 136.

The presence of the high background carrier concentration in sample 135 seems to indicate an unintentional elemental impurity. However, it appears that a more likely cause is an intrinsic

Table 1

Electrical data for bulk InP, bulk InP with a hydrogen plasma and bulk InP with excess hydrogen

\begin{tabular}{llllll}
\hline $\begin{array}{l}\text { Sample } \\
\text { No. }\end{array}$ & $\begin{array}{l}\text { Hy- } \\
\text { drogen }\end{array}$ & $\begin{array}{l}\text { Plas- } \\
\text { ma }\end{array}$ & $\begin{array}{l}\text { Growth } \\
\text { rate } \\
(\mu \mathrm{m} / \mathrm{h})\end{array}$ & $\begin{array}{l}n(77 \mathrm{~K}) \\
\left(\mathrm{cm}^{-3}\right.\end{array}$ & $\begin{array}{l}\mu(77 \mathrm{~K}) \\
\left(\mathrm{cm}^{2} / \mathrm{V} \cdot \mathrm{s}\right)\end{array}$ \\
\hline 134 & No & No & 0.717 & $1.5 \times 10^{15}$ & 57000 \\
135 & Yes & Yes & 0.724 & $6.0 \times 10^{16}$ & 2700 \\
136 & Yes & No & 0.590 & $1.9 \times 10^{15}$ & 65000 \\
\hline
\end{tabular}

crystal defect such as an anti-site defect or a vacancy. After installing the ECR plasma system, hydrogen and nitrogen were flowed into the growth chamber and analyzed with a UTI-100 quadrupole mass spectrometer both with and without igniting a plasma. No contaminants were observed over the range from 1 to $100 \mathrm{amu}$. The energy of the second PL peak corresponds to a carbon acceptor level [9], but the n-type nature of the layer and the relatively high carrier concentration precludes carbon as the unintentional impurity.

The original purpose behind introducing the hydrogen plasma during growth was to prevent the incorporation of unwanted contaminants.

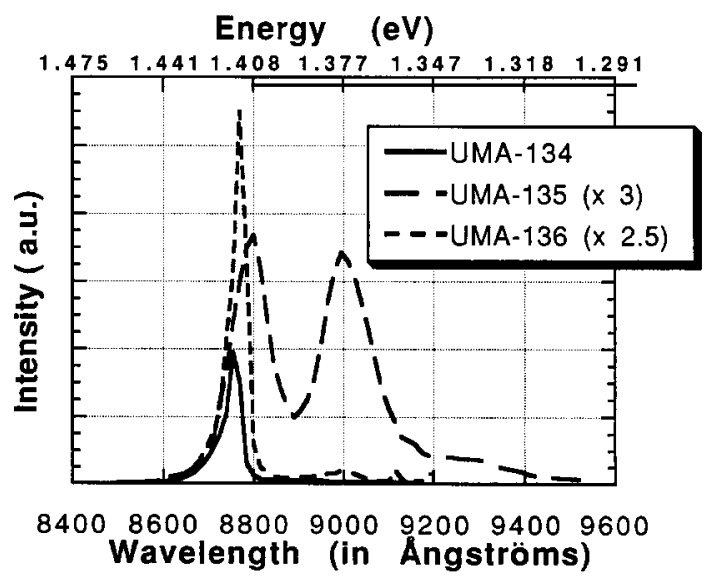

Fig. 3. Photoluminescence spectra of samples 134,135 and 136. The measurements were done at $14 \mathrm{~K}$. 
However, it appears that the hydrogen plasma interfered with the growth process. The atomic hydrogen can tie up surface sites, and can also form volatile compounds on the surface, preventing their incorporation into the growing layer. Reactions between partially decomposed phosphine and the atomic hydrogen would form phosphine, which would not decompose at the substrate. Similar behavior has been seen during hydride vapor phase epitaxy of InP with partially decomposed phosphine [10]. The partially decomposed phosphine would form volatile compounds on the surface, leaving a phosphorus deficient surface. Karlicek et al. [10] have also observed a deterioration in surface quality during growth with a phosphorus deficient surface. Dry etching of InP with hydrogen plasmas results in the formation of indium droplets on the surface $[11,12]$. The formation of indium droplets during etching can even occur at $50^{\circ} \mathrm{C}$, demonstrating the highly reactive nature of the hydrogen plasma. During the growth of InP, the preferential etching of phosphorus by the hydrogen plasma will result in the growth of a phosphorus deficient layer.

Sample 136, grown with excess hydrogen but no plasma, showed only a weak PL signal at 1.378 $\mathrm{eV}$. Since the reactivity of molecular hydrogen is much less than that of atomic hydrogen, the corresponding decrease in the defect level signal was expected. Also the good electrical properties and the excellent surface of sample 136, indicates that it is the high reactivity of the atomic hydrogen that damaged sample 135 . The reduction of the growth rate of sample 136 may be a result of molecular hydrogen tying up surface sites on the growing surface.

Although it is impossible to say with certainty what type of intrinsic crystal defect is responsible for the quality of sample 135 , it does seem that crystal defects and not contamination appears to be the cause of the high carrier concentration, the PL peak at $1.378 \mathrm{eV}$ and the surface degradation.

\section{Conclusions}

An electron cyclotron resonance plasma system has been constructed for the injection of a hydrogen, nitrogen or argon plasma during $\mathrm{CBE}$ growth.
Initial experiments carried out with a hydrogen plasma during the growth of InP resulted in layers with a high n-type carrier concentration and a strong photoluminescence peak at $1.378 \mathrm{eV}$. The most likely cause for this is an intrinsic defect such as an antisite or a vacancy. Although hydrogen plasmas have been effective during the low temperature growth of GaAs, it does not appear that the use of a hydrogen plasma at normal growth temperatures $\left(\approx 500^{\circ} \mathrm{C}\right)$ is useful for $\operatorname{InP}$ growth. Future work will focus on determining the type of crystal defect and developing a better model of the interaction between hydrogen and the growing surface.

\section{Acknowledgments}

The authors would like to thank Mr. Larry Davis for performing the photoluminescence measurements. The authors would also like to acknowledge the assistance provided by both MKS Instruments Inc. and Wavemat, Inc. This work was supported by the US Army Research Office, URI Program, Contract DAAL03-86-K-0007.

\section{References}

[1] S. Sugata, A. Takamori, N. Takado, K. Asakawa, E. Miyauchi and H. Hashimoto, J. Vacuum Sci. Technol. B6 (1988) 1087.

[2] S. Zembutsu and T. Sasaki, J. Crystal Growth 77 (1986) 250.

[3] G.V. Jagannathan, M.L. Andrews and A.T. Habig, Appl. Phys. Letters 56 (1990) 2019.

[4] S. Fujieda, M. Mizuta and Y. Matsumoto, Japan. J. Appl. Phys. 26 (1987) 2067

[5] S. Meikle, H. Nomura, Y. Nakanishi and Y. Hatanaka, J. Appl. Phys. 67 (1990) 483.

[6] E.G. Woelk, M.E. Sherwin and G.O. Munns, J. Crysta] Growth 110 (1991) 452.

[7] Wavemat, Inc., 44780 Helm St. Plymouth, MI 48170 , USA.

[8] J. Asmussen, J. Vacuum Sci. Technol. A7 (1989) 883.

[9] L.D. Zhu, K.T. Chan, D.K. Wagner and J.M. Ballantyne, J. Appl. Phys. 57 (1985) 5486.

[10] R.F. Karlicek, Jr., Mitcham, J.C. Ginocchio and B. Hammarlund, J. Electrochem. Soc. 134 (1987) 470.

[11] R.P.H. Chang, C.C. Chang and S. Darack, J. Vacuum Sci. Technol. 20 (1982) 45.

[12] J.H. Thomas III, G. Kaganowicz and J.W. Robinson, J. Electrochem. Soc. 135 (1988) 1201. 